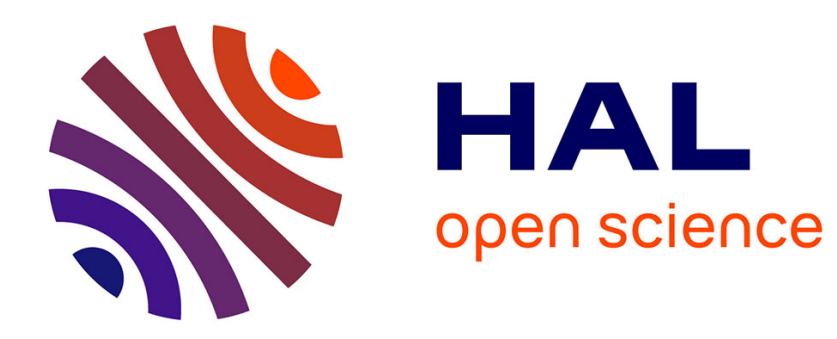

\title{
Fully Nonlinear Global Modes in Spatially Developing Media
}

Benoît Pier, Patrick Huerre

\section{To cite this version:}

Benoît Pier, Patrick Huerre. Fully Nonlinear Global Modes in Spatially Developing Media. Physica D: Nonlinear Phenomena, 1996, 97, pp.206-222. 10.1016/0167-2789(96)00152-2 . hal-00119915

\section{HAL Id: hal-00119915 https://hal.science/hal-00119915}

Submitted on 13 Dec 2006

HAL is a multi-disciplinary open access archive for the deposit and dissemination of scientific research documents, whether they are published or not. The documents may come from teaching and research institutions in France or abroad, or from public or private research centers.
L'archive ouverte pluridisciplinaire HAL, est destinée au dépôt et à la diffusion de documents scientifiques de niveau recherche, publiés ou non, émanant des établissements d'enseignement et de recherche français ou étrangers, des laboratoires publics ou privés. 


\title{
Fully Nonlinear Global Modes in Spatially Developing Media
}

\author{
Benoît Pier and Patrick Huerre \\ Laboratoire d'Hydrodynamique (LadHyX), École polytechnique, \\ F-91128 Palaiseau cedex, France.
}

\begin{abstract}
Global modes on a doubly-infinite one-dimensional domain $-\infty<X<+\infty$ are studied in the context of the complex Ginzburg-Landau equation with slowly spatially varying coefficients. A fully nonlinear frequency selection criterion is derived for globalmode solutions under the assumption of weak inhomogeneity of the medium. The global mode is found to be governed by the fully nonlinear equations in a region of finite size, and by the linearized equations in the vicinity of $X= \pm \infty$. Asymptotic matching techniques are used to relate the WKBJ approximations in the linear and nonlinear regions through appropriate transition layers. The real global frequency is determined by requiring that spatial branches issuing from $X=-\infty$ and $X=+\infty$ be continuously connected at a saddle point of the local nonlinear dispersion relation $\omega=\Omega^{n \ell}\left(k, R^{2}, X\right)$ between the frequency $\omega$, the wavenumber $k$ and amplitude $R$ at a given station $X$. The results constitute a fully nonlinear generalization of the linear frequency selection criteria previously obtained by Chomaz et al. [6], Monkewitz et al. [20], and Le Dizès et al. [16].
\end{abstract}

\section{Introduction}

Our present understanding of hydrodynamic instabilities relies, to a great extent, on the scientific achievements of Fritz Busse and his colleagues. For instance, an intimate knowledge of the Busse balloon [3] has indeed become prerequisite reading to any serious student of Rayleigh-Bénard convection in a fluid layer heated from below, one of the most widely studied closed flow systems. This is just one example of Fritz Busse's fundamental and lasting contributions to hydrodynamics. Fritz has devoted a relatively small portion of his boundless energy to the study of open shear flows, which may explain why our comprehension of instabilities in open flows may have not reached the same fully mature stage.

Preprint submitted to Elsevier Science

22 May 1996 
This contribution, which is dedicated to Fritz Busse in celebration of his 60th birthday, illustrates one possible approach which could be successful in spatially developing open flows exhibiting a global resonance.

Spatially developing flows may, under certain conditions, develop self-sustained oscillations. Fluctuations saturate at a finite amplitude in the locally unstable regions of the flow and become tuned at an overall frequency. The intrinsic frequency and the associated spatial distribution of fluctuations define a global mode. Relatively little is known regarding the nonlinear evolution stage of such objects. Chomaz [4] and Couairon \& Chomaz [7] have recently introduced generalized definitions of absolute, convective and global instability applicable to the fully nonlinear case and demonstrated that linear absolute instability is not always synonymous with global instability. Furthermore, these authors have brought to light the close relationship existing between the dynamical systems point of view and the more classical description in terms of a dispersion relation. The goal of the present study is the derivation of a fully nonlinear global mode selection criterion in the context of the one-dimensional complex Ginzburg-Landau equation with slowly varying coefficients.

The main stability concepts pertaining to spatially developing open flows have been reviewed by Huerre \& Monkewitz [11]. Recent developments have been discussed in Le Dizès et al. [16]. It is here sufficient to recall that specific classes of spatially developing open flows such as bluff-body wakes [18,9,13,24], low density jets $[23,19]$ and counterflow mixing layers [25] may sustain in specific parameter ranges synchronized periodic oscillations over an extended region of the flow. In other words, such systems undergo a Hopf bifurcation as some control parameter is continuously varied. Global mode concepts have precisely been introduced to account for this peculiar behavior of open flows.

In most basic flows of interest, the velocity profile is nonuniform in the streamwise direction, and in order to distinguish between local and global properties it is essential to assume that streamwise variations are slow over a typical instability wavelength. Under this hypothesis, local stability properties are obtained within the parallel flow approximation by freezing the slow streamwise coordinate $X$. At the local level of description, linear stability properties of normal modes $e^{i(k x-\omega t)}$ are then characterized by a local dispersion relation $D(k, \omega, X)=0$ between complex wavenumber $k$ and frequency $\omega$. The goal of a global analysis is to construct in a self-consistent manner a global spatial distribution made up of saturated instability waves governed by the local properties of the flow. The global frequency $\omega_{g}$ is then considered as an eigenvalue to be determined by requiring that perturbations decay at $X= \pm \infty$. The resulting global mode is viewed as a theoretical analogue of the self-sustained oscillatory structures that are observed experimentally. 
The analysis by Chomaz et al. [6] of the linearized Ginzburg-Landau equation with slowly varying coefficients has demonstrated that a region of absolute instability is a necessary condition for linearly unstable global modes to exist. The dominant complex global frequency $\omega_{g}$ is obtained, at leading order in the WKBJ approximation, for a saddle point of the local linear dispersion relation $\omega=\Omega^{\ell}(k, X)$, i.e.,

$$
\omega_{g} \sim \omega_{s}=\Omega^{\ell}\left(k_{s}, X_{s}\right)
$$

where the complex pair $\left(k_{s}, X_{s}\right)$ satisfies

$$
\frac{\partial \Omega^{\ell}}{\partial k}\left(k_{s}, X_{s}\right)=\frac{\partial \Omega^{\ell}}{\partial X}\left(k_{s}, X_{s}\right)=0
$$

The same linear frequency selection criterion has been derived by Monkewitz et al. [20] in the framework of the Navier-Stokes equations linearized around an arbitrary weakly nonparallel basic flow. More recently, the frequency selection criterion pertaining to the Ginzburg-Landau equation over a doubly infinite domain has been examined anew by resorting to a comparison equation method (Le Dizès et al. [16]). It has been shown that global modes are either of type 1 , with two simple turning points in the complex $X$-plane, or type 2 , with a double turning point as defined in (2). The validity of the selection criterion (2) has further been confirmed by the exact Green function calculations of Hunt \& Crighton [12].

The extension of these concepts to the nonlinear régime has until recently appeared somewhat problematic. It seems, at first sight, natural to perform a weakly nonlinear analysis in the vicinity of the global mode onset. Such an approach is straightforward for nonparallel effects of order unity, as shown by Chomaz et al. [5] in the case of the Ginzburg-Landau equation with a linearly or quadratically varying control parameter. Paradoxically, the introduction of a weakly nonparallel assumption in addition to the weakly nonlinear one leads to unexpected difficulties [16]: in the limit of zero nonparallelism, the Landau constant does not converge to a well defined limit of constant sign. In other words, the Landau equation formalism which purportedly describes a Hopf bifurcation near onset does not apply unless drastic conditions are met. In order to circumvent these difficulties, we choose in the present study to jump to the fully nonlinear régime while keeping the weakly nonparallel assumption. Thus we implement a nonlinear WKBJ approximation scheme which remains valid for amplitudes of order unity, as encountered in the phase dynamics of patterns $[21,8,15,17]$ or in the description of slowly varying fully nonlinear wavetrains $[10,2]$.

The main objective of the paper is the generalization of the linear frequency 
selection criterion $(1,2)$ to fully nonlinear global modes provided that the local nonlinear dispersion relation of the weakly inhomogenous flow is given. We should emphasize that, in contrast to the linear criterion, the present formulation will only involve purely real values of the wavenumber, frequency and local streamwise variable. In the present study there is no need to extend analytically the dispersion relation in the complex $k$-plane and $X$-plane, a feature which considerably simplifies the analysis.

The inhomogeneities of the medium are characterized by a small parameter

$$
\epsilon \sim \frac{\lambda}{L} \ll 1
$$

where $L$ is a typical evolution length scale of the basic flow and $\lambda$ a typical wavelength of the instability. Under the assumption $\epsilon \ll 1$, global mode solutions depend on two different length scales: a fast space variable $x$ related to the rapid spatial oscillations of the carrier wave and a slow variable $X=\epsilon x$ describing the slow spatial evolution of the "basic flow". Fluctuations $\Psi(x, t)$ around a given basic state are governed by a partial differential equation, the coefficients of which depend on the slow variable $X$. The local properties of the flow are then recovered by freezing $X$. This separation of scales calls for an analysis in terms of nonlinear WKBJ approximations: the slowly evolving local characteristics may be exploited to construct global mode solutions composed of slowly varying nonlinear wavetrains.

In the course of the study, we are led to distinguish different regions characterizing the global mode spatial structure, as sketched in fig.1. In central nonlinear regions of finite size $\left(\mathrm{CNL}^{ \pm}\right)$the wave has finite amplitude and satisfies the fully nonlinear governing equation of the problem. These central nonlinear regions are surrounded by two semi-infinite outer linear regions $\left(\mathrm{OL}^{ \pm}\right)$where the global mode satisfies the linearized equation and is exponentially decaying towards infinity, as required by the boundary conditions. Transition layers $\left(\mathrm{TL}^{1,2}\right)$ of size $\epsilon^{1 / 2}$ (measured in units of $X$ ) allow a smooth crossover from the central nonlinear regions $\left(\mathrm{CNL}^{ \pm}\right)$into the outer linear regions $\left(\mathrm{OL}^{ \pm}\right)$. As shown later, the existence of a nonlinear global mode requires two wavenumber branches to meet in the core of the nonlinear regions, at a saddle point of the local nonlinear dispersion relation. An additional internal layer (SP) of size $\epsilon^{1 / 2}$ around this saddle point needs to be introduced in order to generate the nonlinear frequency selection criterion at arbitrary order. The selection mechanism is therefore entirely determined by the saddle point structure. Thus this inner transition layer (SP) is surrounded by nonlinear regions of finite extent $\left(\mathrm{CNL}^{ \pm}\right)$which in turn are connected through transition layers $\left(\mathrm{TL}^{1,2}\right)$ to the outer linear regions $\left(\mathrm{OL}^{ \pm}\right)$extending towards infinity. 


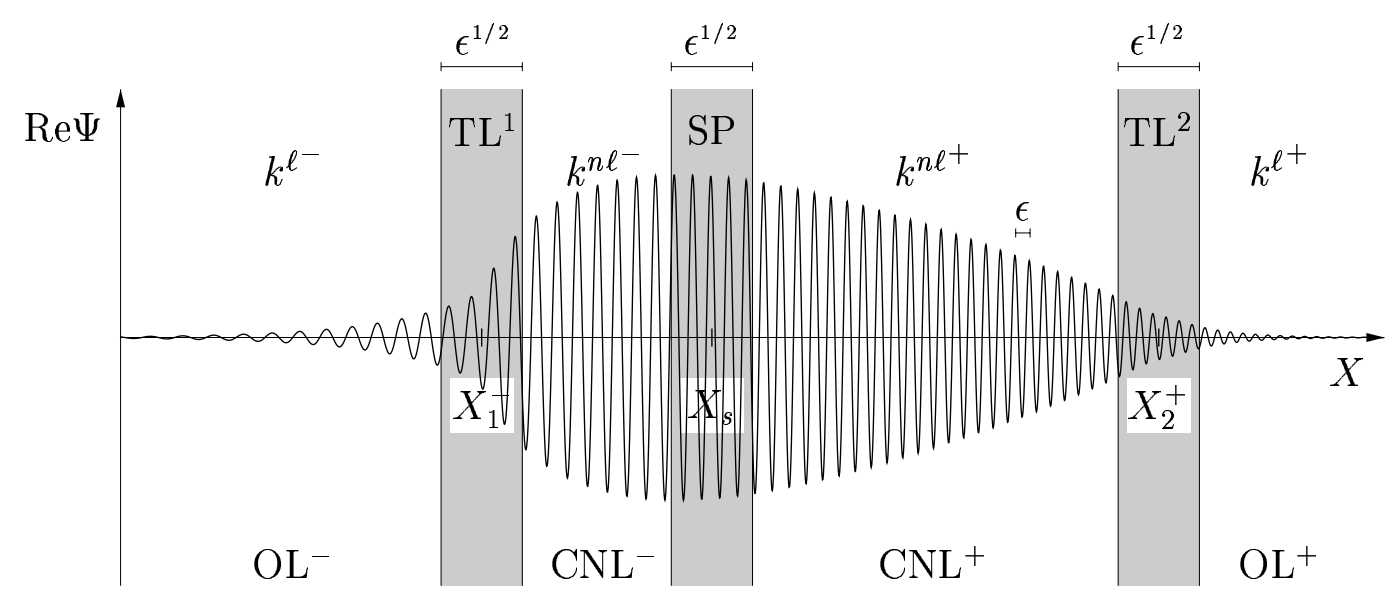

Fig. 1. Fully nonlinear global mode spatial structure: $\mathrm{OL}^{ \pm}$, outer semi-infinite linear regions close to $X= \pm \infty$; $\mathrm{TL}^{1,2}$, transition layers of thickness $O\left(\epsilon^{1 / 2}\right)$ connecting linear and nonlinear regions; $\mathrm{CNL}^{ \pm}$, central nonlinear regions of size $O(1)$; SP, saddle-point region of thickness $O\left(\epsilon^{1 / 2}\right)$.

The organization of the present paper reflects the global mode structure. The global mode problem is stated in $\S 2$ and the linear WKBJ approximations pertaining to the outer regions $\left(\mathrm{OL}^{ \pm}\right)$are recalled in $\S 3$. Corresponding nonlinear WKBJ solutions relevant to the central nonlinear regions $\left(\mathrm{CNL}^{ \pm}\right)$are derived in $\S 4$. In $\S 5$ the leading order nonlinear frequency selection criterion is obtained in the following form: a global mode can only be constructed if two wavenumber branches meet at a saddle point in the bulk of the nonlinear regions. The structure of the transition layers connecting linear and nonlinear regions is analyzed in $§ 6$. In closing we summarize the main conclusions and address some future developments.

\section{Nonlinear Eigenvalue Problem}

Throughout the study, complex scalar fluctuations $\Psi(x, t)$ around a given basic state are assumed to be governed by a nonlinear equation of the form

$$
i \frac{\partial \Psi}{\partial t}=\Omega^{n \ell}\left(-i \frac{\partial}{\partial x},|\Psi|^{2}, X\right) \Psi
$$

where $X=\epsilon x$ is a slow space variable which describes the inhomogeneities of the medium and $\epsilon$ is the WKBJ parameter introduced in (3). The operator $\Omega^{n \ell}$ is the physical space counterpart of the local nonlinear dispersion relation $\omega=$ $\Omega^{n \ell}\left(k, R^{2}, X\right)$ to be defined later. 
A nonlinear global mode is then defined as a time-periodic solution of eq.(4) of real frequency $\omega$, satisfying vanishing boundary conditions at infinity. Thus it can be written as

$$
\Psi(x, t)=\psi(x, \omega) e^{-i \omega t}
$$

where the global frequency $\omega$ and the amplitude $\psi$ are governed by the differential equation

$$
\left[\omega-\Omega^{n \ell}\left(-i \frac{\partial}{\partial x},|\psi|^{2}, X\right)\right] \psi=0
$$

with the boundary conditions

$$
\lim _{x \rightarrow \pm \infty} \psi(x, \omega)=0
$$

In the particular case of the complex Ginzburg-Landau equation [11],

$$
\begin{aligned}
i \frac{\partial \Psi}{\partial t}=\left(\omega_{0}(X)+\frac{1}{2} \omega_{k k}(X) k_{0}(X)^{2}\right) \Psi & +i \omega_{k k}(X) k_{0}(X) \frac{\partial \Psi}{\partial x} \\
& -\frac{1}{2} \omega_{k k}(X) \frac{\partial^{2} \Psi}{\partial x^{2}}+\gamma(X)|\Psi|^{2} \Psi
\end{aligned}
$$

the nonlinear dispersion operator $\Omega^{n \ell}$ may be separated into linear and nonlinear parts as

$$
\Omega^{n \ell}\left(-i \frac{\partial}{\partial x},|\Psi|^{2}, X\right) \equiv \Omega^{\ell}\left(-i \frac{\partial}{\partial x}, X\right)+\gamma(X)|\Psi|^{2},
$$

where the linear dispersion operator $\Omega^{\ell}$ reads

$$
\begin{aligned}
\Omega^{\ell}\left(-i \frac{\partial}{\partial x}, X\right) \equiv \omega_{0}(X) & +\frac{1}{2} \omega_{k k}(X) k_{0}(X)^{2} \\
& +i \omega_{k k}(X) k_{0}(X) \frac{\partial}{\partial x}-\frac{1}{2} \omega_{k k}(X) \frac{\partial^{2}}{\partial x^{2}} .
\end{aligned}
$$

The complex functions $\omega_{0}(X), \omega_{k k}(X), k_{0}(X)$, and $\gamma(X)$ which solely depend on the slow space variable $X$ account for the weak inhomogeneities of the medium and their meaning is discussed in $\S 3$. 
The goal of the present study is to solve the nonlinear eigenvalue problem $(5,6)$ where the global mode amplitude $\psi$ is $O(1)$ in an interval of finite extent. We construct such global mode solutions in the régime $\epsilon \ll 1$ and determine how the global frequencies $\omega_{g}$ depend on the characteristics of the "basic flow", as specified by the nonlinear dispersion relation.

Since global modes must decay at infinity, they are governed by the linearized equation for sufficiently large $X$. The solutions of the associated linear equation are therefore required in outer linear regions $\left(\mathrm{OL}^{ \pm}\right)$, as reviewed in the next section.

\section{Outer Linear WKBJ Regions $\mathrm{OL}^{ \pm}$}

In the present section we examine the structure of the outer linear regions $\left(\mathrm{OL}^{ \pm}\right)$ in fig. 1 and recall the main features of the solutions of the linearized equation

$$
i \frac{\partial \Psi}{\partial t}=\Omega^{\ell}\left(-i \frac{\partial}{\partial x}, X\right) \Psi
$$

for an arbitrary frequency $\omega$ of order unity, as studied by Chomaz et al. [6] and Le Dizès et al. [16].

Upon substituting $\partial / \partial x \rightarrow i k$ and $\partial / \partial t \rightarrow-i \omega$ in $(8,9)$ one obtains the associated local linear dispersion relation of the Ginzburg-Landau model in the form

$$
\omega=\Omega^{\ell}(k, X) \equiv \omega_{0}(X)+\frac{1}{2} \omega_{k k}(X)\left(k-k_{0}(X)\right)^{2} .
$$

In order to enforce causality we assume throughout that sufficiently large wavenumbers are damped, i.e., $\omega_{k k, i}(X) \equiv \operatorname{Im}\left[\omega_{k k}(X)\right]<0$ for all $X$. According to (10) the frequency $\omega$ is a simple quadratic function of the complex wavenumber $k$. Thus there exist for each value of the frequency two linear spatial branches given by

$$
k^{\ell^{ \pm}}(X, \omega) \equiv k_{0}(X) \pm \sqrt{2 \frac{\omega-\omega_{0}(X)}{\omega_{k k}(X)}} .
$$

Under the assumption $\omega_{k k, i}(X)<0$ for all $X$, the square root branch in (11) can always be chosen such that $k_{i}^{\ell^{+}}(X)>0$ and $k_{i}^{\ell^{-}}(X)<0$ for large enough $\omega$. The introduction of the functions $k_{0}(X)$ and $\omega_{0}(X)$ may now be justified: at each 
station $X, k_{0}$ and $\omega_{0}$ denote the complex absolute wavenumber and frequency defined by $\frac{\partial \Omega^{\ell}}{\partial k}\left(k_{0}, X\right)=0, \omega_{0}=\Omega^{\ell}\left(k_{0}, X\right)$, as extensively discussed in [11]. In other words, $\omega_{0}(X)$ is a branch point of the function $k^{\ell}(X, \omega)$ in the complex $\omega$-plane, as clearly displayed in (11).

Under the slowly varying medium hypothesis $(\epsilon \ll 1)$ a solution of eq.(9) with frequency $\omega$ can be obtained in terms of WKBJ approximations [1]. The spatial behavior of the solution is described by a rapidly varying phase, corresponding to the local wavenumber, and a slowly varying envelope which, for a given value of the frequency $\omega$, read

$$
\psi^{\ell}(X, \omega)=A^{\ell}(X, \omega) \exp \frac{i}{\epsilon} \int^{X} k^{\ell}(s, \omega) d s
$$

where $k^{\ell}(X, \omega)$ is one of the spatial branches $(11)$.

In classical WKBJ fashion, the envelope ${ }^{1} A^{\ell}(X)$ is expanded in powers of $\epsilon$

$$
A^{\ell}(X) \sim A_{0}^{\ell}(X)+\epsilon A_{1}^{\ell}(X)+\epsilon^{2} A_{2}^{\ell}(X)+\ldots
$$

and the spatial derivative operator is decomposed into

$$
\partial_{x}=i k^{\ell}(X)+\epsilon \partial_{X}
$$

Upon substituting $(12,13,14)$ into the governing equation $(9)$, one obtains

$$
\omega\left(A_{0}^{\ell}+\epsilon A_{1}^{\ell}+\ldots\right)=\Omega^{\ell}\left(k^{\ell}(X)-i \epsilon \partial_{X}, X\right)\left(A_{0}^{\ell}+\epsilon A_{1}^{\ell}+\ldots\right) .
$$

Note that differentiation with respect to the fast variable, i.e., multiplication by $i k^{\ell}(X)$, does not commute with the slow derivative operator $\partial_{X}$ [14]. Thus $\Omega^{\ell}\left(k^{\ell}(X)-i \epsilon \partial_{X}\right)$ admits the expansion

$$
\begin{aligned}
\Omega^{\ell}\left(k^{\ell}(X)-i \epsilon \partial_{X}\right) & =\Omega^{\ell}-i \epsilon \frac{1}{2}\left(\Omega_{k}^{\ell} \partial_{X}+\partial_{X} \Omega_{k}^{\ell}\right)+O\left(\epsilon^{2}\right) \\
& =\Omega^{\ell}-i \epsilon\left(\Omega_{k}^{\ell} \partial_{X}+\frac{1}{2} \Omega_{k k}^{\ell} k_{X}^{\ell}(X)\right)+O\left(\epsilon^{2}\right) .
\end{aligned}
$$

On the r.h.s. of these equations, $\Omega^{\ell}$ and its derivatives with respect to $k$ are evaluated at $k^{\ell}(X)$. In eq.(15) the slow derivative operator $\partial_{X}$ only applies to the wavefunction, hence to $k^{\ell}(X)$, but not to the second argument $X$ of $\Omega^{\ell}(k, X)$.

\footnotetext{
${ }^{1}$ For simplicity we no longer explicitly mention the frequency dependence.
} 
At lowest order, eq.(15) reduces to the dispersion relation (10), which yields the local wavenumber $k^{\ell}(X)$ in terms of $\omega$, as given by (11).

At order $\epsilon$, we obtain

$$
\left[\omega-\Omega^{\ell}\left(k^{\ell}(X), X\right)\right] A_{1}^{\ell}=-i \Omega_{k}^{\ell}(X) \frac{d A_{0}^{\ell}}{d X}-\frac{i}{2} \Omega_{k k}^{\ell}(X) k_{X}^{\ell}(X) A_{0}^{\ell}
$$

with the notations

$$
\Omega_{k}^{\ell}(X)=\frac{\partial \Omega^{\ell}}{\partial k}\left(k^{\ell}(X), X\right) \quad \text { and } \quad \Omega_{k k}^{\ell}(X)=\frac{\partial^{2} \Omega^{\ell}}{\partial k^{2}}\left(k^{\ell}(X), X\right) .
$$

By invoking the local dispersion relation (10), one obtains the obvious solvability condition for $A_{0}^{\ell}$

$$
\frac{d A_{0}^{\ell}}{d X}=-\frac{1}{2} k_{X}^{\ell}(X) \frac{\Omega_{k k}^{\ell}(X)}{\Omega_{k}^{\ell}(X)} A_{0}^{\ell}(X)
$$

Thus, at first order, the solution of the linearized equation reads

$$
\psi^{\ell}(X) \sim A_{0}^{\ell}\left(X_{1}\right) \exp \left(-\frac{1}{2} \int_{X_{1}}^{X} k_{X}^{\ell}(s) \frac{\Omega_{k k}^{\ell}(s)}{\Omega_{k}^{\ell}(s)} d s\right) \exp \left(\frac{i}{\epsilon} \int_{X_{1}}^{X} k^{\ell}(s) d s\right)
$$

where $X_{1}$ is some arbitrarily specified reference point.

The linear Ginzburg-Landau equation admits two spatial branches $k^{\ell^{ \pm}}(X)$ given by (11); thus eq.(17) yields two WKBJ approximations $\psi^{\ell^{ \pm}}(X)$ for two linearly independent solutions of eq.(9). Let us assume that $k_{i}^{\ell^{+}}(X)>0$ and $k_{i}^{\ell^{-}}(X)<0$ for sufficiently large $|X|$ (for a discussion of the relationship between this assumption and causality see [16]). Under this assumption the WKBJ approximation $\psi^{\ell^{-}}(X)$ is subdominant when $X \rightarrow-\infty$, i.e., exponentially small with respect to $\psi^{\ell^{+}}(X)$; similarly the solution $\psi^{\ell^{+}}(X)$ becomes subdominant for $X \rightarrow+\infty$. Boundary conditions (6) require the choice of $\psi^{\ell^{ \pm}}(X)$ for $X \rightarrow \pm \infty$. A global mode solution is thus solely composed of the branch $k^{\ell^{-}}$for $X \rightarrow-\infty$ and of the branch $k^{\ell^{+}}$for $X \rightarrow+\infty$. In the following sections a nonlinear solution is derived which allows a smooth crossover from one linear branch to the other as $X$ varies from $-\infty$ to $+\infty$. 


\section{Central Nonlinear wKBJ Regions $\mathrm{CNL}^{ \pm}$}

In this section a fully nonlinear solution of equation (4) is constructed in the central regions $\left(\mathrm{CNL}^{ \pm}\right)$of fig.1 by means of the nonlinear dispersion relation, for an arbitrary frequency of order unity.

Let us first consider in the same notations as eq.(4) the homogenous nonlinear equation with constant coefficients

$$
i \frac{\partial \Psi}{\partial t}=\Omega^{n \ell}\left(-i \frac{\partial}{\partial x},|\Psi|^{2}\right) \Psi .
$$

This equation admits nonlinear travelling wave Stokes solutions of the form

$$
\Psi(x, t)=R e^{i(k x-\omega t)},
$$

where the amplitude $R$, wavenumber $k$, and frequency $\omega$ are real parameters bound by the nonlinear dispersion relation

$$
\omega=\Omega^{n \ell}\left(k, R^{2}\right) .
$$

This complex equation completely defines the family of solutions (18). It is also convenient to introduce a reduced nonlinear dispersion relation as follows.

Taking the imaginary part of complex equation (19) shows that the amplitude $R^{2}$ is slaved to the wavenumber $k$ and is implicitly given by

$$
0=\Omega_{i}^{n \ell}\left(k, R^{2}\right) .
$$

Replacing the amplitude $R^{2}$ as a function of $k$ in the real part of (19),

$$
\omega=\Omega_{r}^{n \ell}\left(k, R^{2}\right),
$$

yields the reduced nonlinear dispersion relation in the form

$$
\omega=\Omega^{N L}(k) \equiv \Omega_{r}^{n \ell}\left(k, R^{2}(k)\right) .
$$

This reduced form clearly demonstrates that Stokes travelling waves (18) indeed constitute a one-parameter family of finite amplitude solutions, of real parameter $k$. 
In the context of the complex Ginzburg-Landau model (7), the complete nonlinear dispersion relation reads

$$
\omega=\Omega^{n \ell}\left(k, R^{2}\right) \equiv \omega_{0}+\frac{1}{2} \omega_{k k}\left(k-k_{0}\right)^{2}+\gamma R^{2}=\Omega^{\ell}(k)+\gamma R^{2},
$$

and the reduced dispersion relation may explicitely be determined by following the procedure outlined above. Extracting $R^{2}$ from the imaginary part of (20), one finds

$$
R^{2}=\frac{\operatorname{Im}\left[\Omega^{\ell}(k)\right]}{\operatorname{Im}\left[\gamma^{\star}\right]}
$$

and by substitution into the real part of (20), one readily obtains the reduced nonlinear dispersion relation

$$
\omega=\Omega^{N L}(k)=\frac{\operatorname{Im}\left[\gamma^{\star} \Omega^{\ell}(k)\right]}{\operatorname{Im}\left[\gamma^{\star}\right]} .
$$

Since (22) is quadratic in $k$, to each frequency $\omega$ are associated, under suitable conditions, two nonlinear spatial branches $k^{n \ell^{ \pm}}(\omega)$, and, via (21), two amplitudes $\mathcal{R}^{2^{ \pm}}(\omega)$. Thus the complete nonlinear dispersion relation (20) is seen to possess two pairs of solutions

$$
\left(k, R^{2}\right)=\left(k^{n \ell^{ \pm}}(\omega), \mathcal{R}^{2 \pm}(\omega)\right) .
$$

Such pairs of solutions only exist if (20) can be solved for real $k$ and real nonnegative $R^{2}$. For given coefficients in (20) these conditions correspond to a range of allowable frequencies.

In a weakly inhomogenous medium where the coefficients of the nonlinear equation are now functions of the slow space variable $X$ (equations $(4,7)$ ), a local nonlinear dispersion relation may still be derived of the form

$$
\begin{aligned}
\omega=\Omega^{n \ell}\left(k, R^{2}, X\right) & \equiv \omega_{0}(X)+\frac{1}{2} \omega_{k k}(X)\left(k-k_{0}(X)\right)^{2}+\gamma(X) R^{2} \\
& =\Omega^{\ell}(k, X)+\gamma(X) R^{2}
\end{aligned}
$$

and the corresponding local reduced nonlinear dispersion relation may be formally expressed as

$$
\omega=\Omega^{N L}(k, X) \equiv \frac{\operatorname{Im}\left[\gamma^{\star}(X) \Omega^{\ell}(k, X)\right]}{\operatorname{Im}\left[\gamma^{\star}(X)\right]} .
$$



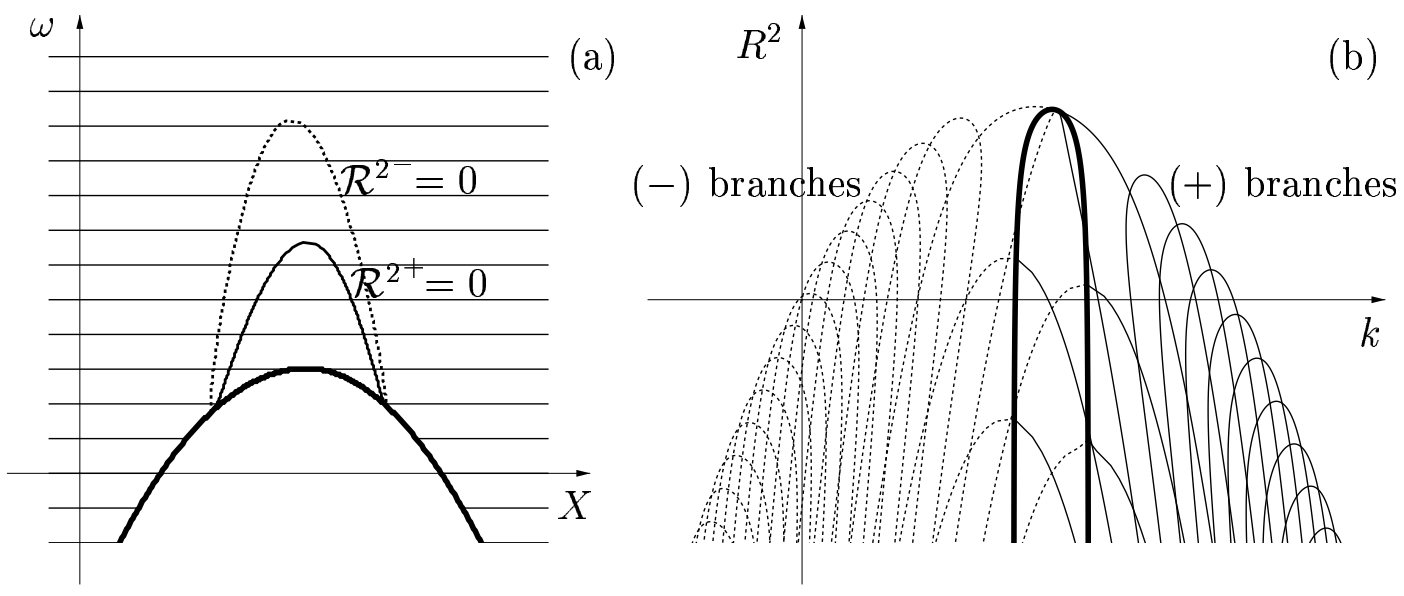

Fig. 2. Nonlinear spatial branches in the $X-\omega$ and $k-R^{2}$ planes. In (a) the horizontal lines $\omega=$ const. indicate in the $X$ - $\omega$ plane the allowable domain, delineated by the bold critical curve, where the nonlinear dispersion relation (23) admits real solutions in the $k-R^{2}$ plane sketched in (b). Each of the lines $\omega=$ const. is mapped into two spatial branches $\left(k^{n \ell^{ \pm}}(X), \mathcal{R}^{2^{ \pm}}(X)\right)$ in the $k-R^{2}$ plane: the $(+)$ branches are represented by solid curves, the (-) branches by dotted curves. For frequencies such that the line $\omega=$ const. intersects the boundary of the allowable domain (bold curve in (a)), the two branches in the $k-R^{2}$ plane meet at the corresponding image critical curve (bold curve in (b)). The subregions of the allowable domain corresponding to $R^{2}>0$ are delimited in (a) by the curves $\mathcal{R}^{2+}(X, \omega)=0$ (solid) and $\mathcal{R}^{2-}(X, \omega)=0$ (dotted).

Local wavenumber and amplitude are obtained in the same way as in the homogenous case; they are real functions of the frequency and the slow space variable:

$$
\left(k, R^{2}\right)=\left(k^{n \ell^{ \pm}}(X, \omega), \mathcal{R}^{2^{ \pm}}(X, \omega)\right)
$$

solutions of (23) and (24).

Let us now examine the properties of the double-valued mapping between the $X-\omega$ and $k-R^{2}$ planes defined by solution pairs (25). The $X-\omega$ plane separates into an allowable region where two real solution pairs (25) satisfy the local nonlinear dispersion relation (23) and a region where no such real solutions are obtained (fig.2-a). For given $X$, real wavenumber solutions of the quadratic equation (24) are found to exist when $\omega \rightarrow+\infty(-\infty)$ if $\omega_{k k, r}-\omega_{k k, i} \gamma_{r} / \gamma_{i}>0(<0)$. Let us assume that $\omega_{k k, r}(X)-\omega_{k k, i}(X) \gamma_{r}(X) / \gamma_{i}(X)>0$ for all $X$. As $\omega$ decreases for a given $X$, the two pairs merge at a critical value of $\omega$ when the discriminant of the quadratic equation (24) vanishes. The set of such points in the $X$ - $\omega$ plane defines the critical line (bold curve of fig.2-a), i.e., the boundary of the allowable domain indicated by the hatched region of fig.2-a. Below this critical line the nonlinear dispersion relations (23) or (24) admit no real solutions. Each point $(X, \omega)$ of the allowable domain then maps into two points in the $k-R^{2}$ plane (fig.2-b) through 
the mapping functions (25). A solution of real uniform frequency is in fact represented in the $X-\omega$ plane by a straight horizontal line $\omega=$ const. As $X$ varies for a given $\omega$, solutions of eq. (25) move along two distinct branches $\left(k^{n \ell^{+}}(X), \mathcal{R}^{2^{+}}(X)\right)$ and $\left(k^{n \ell^{-}}(X), \mathcal{R}^{2-}(X)\right)$ in the $k-R^{2}$ plane, as indicated by the continuous and dashed curves in fig.2-b. If the line $\omega=$ const. is high enough and does not meet the critical line delimiting the allowable domain in the $X$ - $\omega$ plane, the two corresponding branches in the $k-R^{2}$ plane do not intersect; if by contrast the horizontal line $\omega=$ const. is not entirely contained in the allowable hatched domain, spatial branches in the $k-R^{2}$ plane are not defined for the portion of the line situated outside the allowable domain. In fact, upon hitting the critical line in the $X$ - $\omega$ plane, $(+)$ and $(-)$ branches in the $k-R^{2}$ plane meet along the image of the critical line (bold curve of fig.2-b) and cease to exist.

Note also that each branch of (25) has physical meaning only if $R^{2} \geq 0$. Thus the subregions of the allowable domain, where meaningful nonlinear solutions are obtained, are further restricted to $\mathcal{R}^{2 \pm}(X, \omega) \geq 0$. These regions are located within the curves labelled $\mathcal{R}^{2+}=0$ and $\mathcal{R}^{2^{-}}=0$ on fig.2-a. If one assumes $\gamma_{i}(X)<0$ uniformly in $X$ (locally supercritical behavior) the condition $\mathcal{R}^{2^{ \pm}}(X, \omega) \geq 0$ corresponds to linearly unstable wavenumbers $k^{n \ell^{ \pm}}(X, \omega)$, as seen by inspection of $(21)$. Elsewhere in the $X-\omega$ plane, $\mathcal{R}^{2^{ \pm}}(X, \omega)<0$, nonlinear solutions pertaining to $(+)$ or $(-)$ branches do not exist and only linear solutions are allowed.

As in the outer linear regions, the spatial distribution is taken to be a rapidly oscillating wave modulated by a slowly varying envelope

$$
\psi^{n \ell}(X)=A^{n \ell}(X) \exp \frac{i}{\epsilon} \int^{X} k^{n \ell}(s) d s .
$$

Upon expanding the envelope in powers of $\epsilon$

$$
A^{n \ell}(X) \sim A_{0}^{n \ell}(X)+\epsilon A_{1}^{n \ell}(X)+\epsilon^{2} A_{2}^{n \ell}(X)+\ldots
$$

interpreting the spatial derivatives as

$$
\partial_{x}=i k^{n \ell}(X)+\epsilon \partial_{X}
$$

and substituting these expansions into eq.(4), one readily obtains the nonlinear counterpart of (15), i.e.,

$$
\begin{array}{r}
\omega\left(A_{0}^{n \ell}+\epsilon A_{1}^{n \ell}+\ldots\right)=\Omega^{n \ell}\left(k^{n \ell}(X)-i \epsilon \partial_{X},\left|A_{0}^{n \ell}+\epsilon A_{1}^{n \ell}+\ldots\right|^{2}, X\right) \\
\left(A_{0}^{n \ell}+\epsilon A_{1}^{n \ell}+\ldots\right) .
\end{array}
$$


At lowest order, we recover the local nonlinear dispersion relation (23), i.e.,

$$
\omega=\Omega^{n \ell}\left(k^{n \ell}(X),\left|A_{0}^{n \ell}(X)\right|^{2}, X\right)
$$

which determines the local wavenumber $k^{n \ell}(X)$ and the leading order amplitude $\left|A_{0}^{n \ell}(X)\right|^{2}=\mathcal{R}^{2}(X)$ as functions of the slow space variable $X$ for a given frequency $\omega$. Note that the phase $\theta_{0}(X)$ of $A_{0}^{n \ell}(X)$ is as yet undetermined.

After expanding the operator $\Omega^{n \ell}$ in powers of $\epsilon$ in the same fashion as in (16), the $\epsilon$ terms of $(27)$ yield

$$
\left(\omega-\Omega^{n \ell}\right) A_{1}^{n \ell}=-i \Omega_{k}^{n \ell} \frac{d A_{0}^{n \ell}}{d X}-\frac{i}{2} \Omega_{k k}^{n \ell} k_{X}^{n \ell} A_{0}^{n \ell}+\Omega_{R^{2}}^{n \ell}\left(A_{0}^{n \ell^{\star}} A_{1}^{n \ell}+A_{1}^{n \ell^{\star}} A_{0}^{n \ell}\right) A_{0}^{n \ell},
$$

where $\Omega^{n \ell}$ and its partial derivatives are evaluated at $\left(k^{n \ell}(X), \mathcal{R}^{2}(X), X\right)$. In the following, $\Omega^{n \ell}(X)$ stands for $\Omega^{n \ell}\left(k^{n \ell}(X), \mathcal{R}^{2}(X), X\right)$.

Upon exploiting the identity $\omega=\Omega^{n \ell}(X)$, the fact that $\frac{d A_{0}^{n \ell}}{d X}=\left(i \frac{d \theta_{0}}{d X}+\frac{\mathcal{R}_{X}^{2}}{2 \mathcal{R}^{2}}\right) A_{0}^{n \ell}$, and the real nature of $A_{0}^{n \ell^{\star}} A_{1}^{n \ell}+A_{1}^{n \ell^{\star}} A_{0}^{n \ell}$, we readily obtain a solvability condition for $\theta_{0}(X)$ of the form

$$
\frac{d \theta_{0}}{d X}=\frac{1}{2} \frac{\operatorname{Re}\left[\Omega_{R^{2}}^{n \ell}(X)^{\star} \Omega_{k}^{n \ell}(X)\right]}{\operatorname{Im}\left[\Omega_{R^{2}}^{n \ell}(X)^{\star} \Omega_{k}^{n \ell}(X)\right]} \frac{\mathcal{R}_{X}^{2}(X)}{\mathcal{R}^{2}(X)}-\frac{1}{2} \frac{\operatorname{Re}\left[\Omega_{R^{2}}^{n \ell}(X)^{\star} \Omega_{k k}^{n \ell}(X)\right]}{\operatorname{Im}\left[\Omega_{R^{2}}^{n \ell}(X)^{\star} \Omega_{k}^{n \ell}(X)\right]} k_{X}^{n \ell}(X) .
$$

Equations $(26,28,29)$ entirely specify the nonlinear solution at leading order.

\section{Saddle Point Region SP: Leading-Order Nonlinear Frequency Se- lection Criterion}

In this section the global frequency $\omega_{g}$ is obtained at lowest order by matching the nonlinear spatial branches $k^{n \ell^{+}}(X)$ and $k^{n \ell^{-}}(X)$.

Solving the linear dispersion relation (10) yields the local complex wavenumbers $k^{\ell^{ \pm}}(X, \omega)$ as functions of the frequency at any location. By contrast the nonlinear dispersion relation (23) admits solution pairs $\left(k^{n \ell^{ \pm}}(X, \omega), \mathcal{R}^{2^{ \pm}}(X, \omega)\right)$ only in the allowable region of the $X-\omega$ plane. Moreover only nonlinear solutions with $\mathcal{R}^{2^{ \pm}}(X, \omega)>0$ have physical meaning.

The relationship between linear and nonlinear spatial branches as a function of frequency is illustrated in fig. 3 . In the $X-\omega$ plane a solution of uniform frequency 

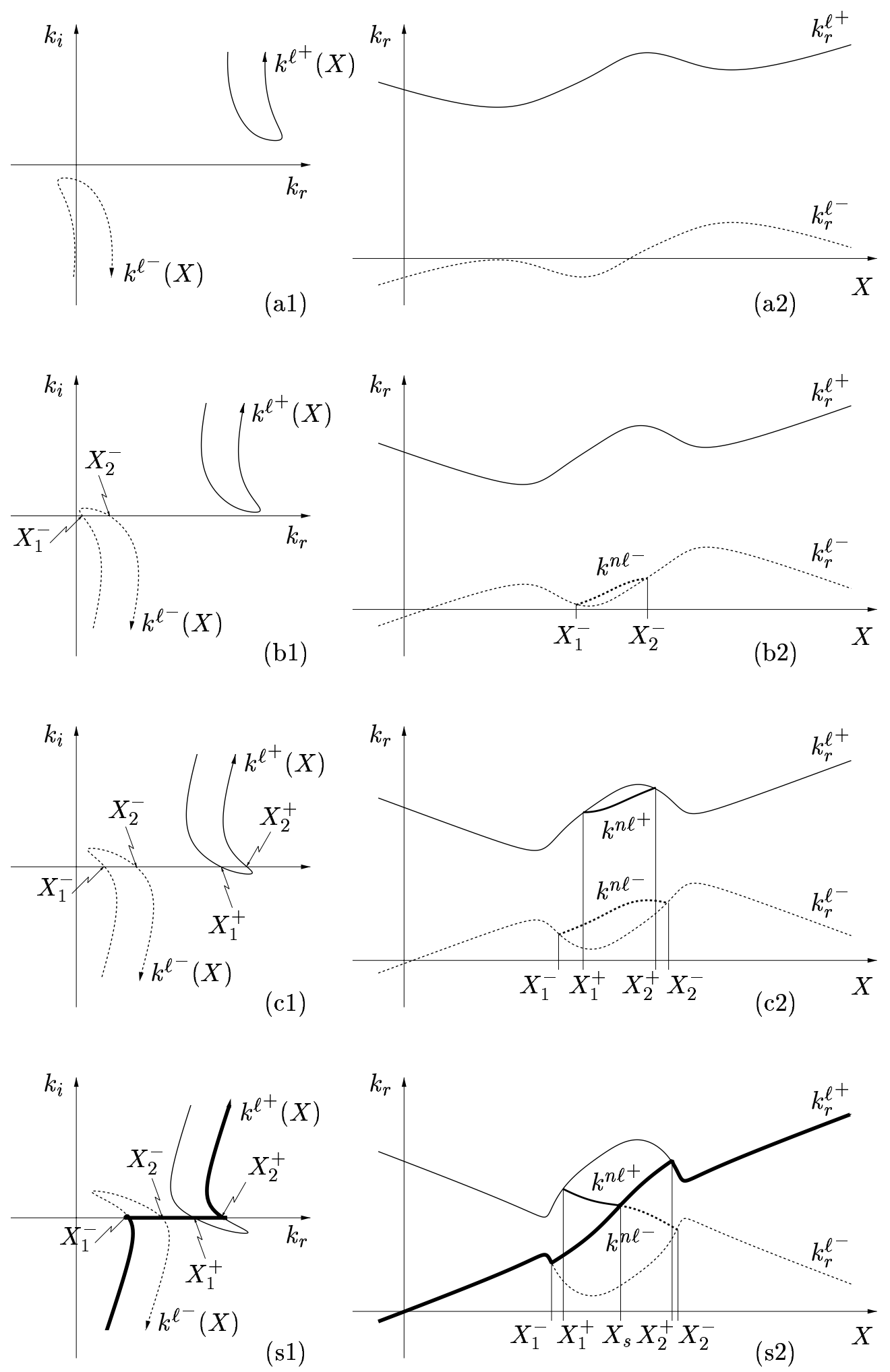


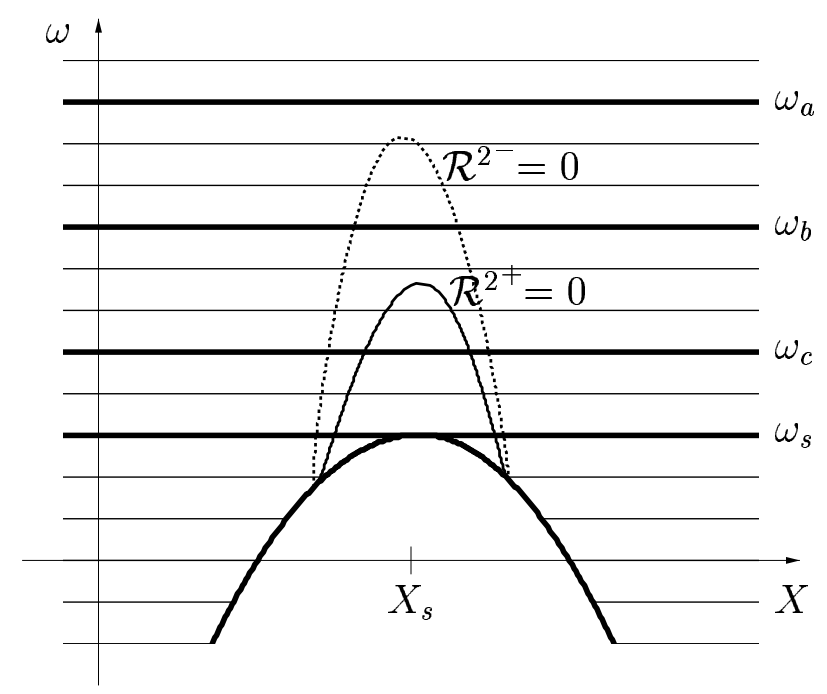

Fig. 3. Relationship between linear and nonlinear spatial branches for different values of the frequency. For a large frequency $\omega_{a}$, the line $\omega=\omega_{a}$ does not intersect the regions $\mathcal{R}^{2^{ \pm}}(X, \omega) \geq 0$ in the $X$ - $\omega$ plane, the linear branches $k^{\ell^{ \pm}}(X)$ do not cross the $k_{r}$-axis in the complex $k$-plane (a1), and nonlinear branches do not exist; the real parts $k_{r}^{\ell^{ \pm}}(X)$ are represented in (a2). For $\omega_{b}$ such that the line $\omega=\omega_{b}$ intersects the region $\mathcal{R}^{2^{-}}(X, \omega) \geq 0$ in the $X-\omega$ plane, the branch $k^{\ell^{-}}(X)$ crosses the $k_{r}$-axis for $X=X_{1}^{-}$ and $X=X_{2}^{-}$in (b1); as a result, the nonlinear $k^{n \ell^{-}}(X)$ exists for $X_{1}^{-}<X<X_{2}^{-}$and is connected to $k_{r}^{\ell^{-}}(X)$ in (b2). The line $\omega=\omega_{c}$ intersects both regions $\mathcal{R}^{2 \pm}(X, \omega)$, thus both linear branches cross the $k_{r}$-axis in (c1); both nonlinear branches exist in the ranges $X_{1}^{ \pm}<X<X_{2}^{ \pm}$respectively (c2). When the frequency equals $\omega_{s}$ such that the line $\omega=\omega_{s}$ is tangent to the boundary of the allowable domain at $X_{s}$ in the $X$ - $\omega$ plane, the nonlinear branches meet at $X_{s}(\mathrm{~s} 2)$, and the crossover between branches is possible; the global spatial branch selected by the global mode is indicated in bold in (s1,2).

is represented by the line $\omega=$ const. This line is mapped, in the complex $k$-plane, into two linear spatial branches $k^{\ell^{ \pm}}(X)$ defined in (11).

Let us recall that, for large enough frequencies $\omega=\omega_{a}$ (see $X$ - $\omega$ plane of fig.3), the linear branches do not cross the $k_{r}$-axis in the complex $k$-plane as long as $\omega_{k k, i}(X)<0$ for all $X$ (fig.3-a1), and that $k^{\ell^{ \pm}}(X)$ denotes the linear branch located in the $k_{i}>0$ and $k_{i}<0$ half-planes respectively. Corresponding variations of $k_{r}^{\ell^{ \pm}}$with $X$ are displayed on fig.3-a2.

When the frequency is decreased to $\omega=\omega_{b}$ (see $X$ - $\omega$ plane of fig.3), the linear branches move about in the complex $k$-plane. The branch $k^{\ell^{ \pm}}(X)$ crosses the $k_{r}$-axis (fig.3-b1) whenever the frequency is such that the line $\omega=\omega_{b}$ intersects, in the $X-\omega$ plane, the region $\mathcal{R}^{2 \pm}(X, \omega)>0$. Indeed a local solution of the nonlinear dispersion relation with $R^{2}=0$ is precisely a solution of the linear dispersion relation, and this linear solution happens to have a real wavenumber. 
Thus at locations where $R^{2}=0$, linear and nonlinear branches meet. Let us denote by $k^{n \ell^{ \pm}}(X, \omega)$ the nonlinear branches that match to the corresponding linear branches $k^{\ell^{ \pm}}(X, \omega)$. In the specific case of fig.3-b, the line $\omega=\omega_{b}$ is partly contained within the region $\mathcal{R}^{2^{-}}>0$ of the $X-\omega$ plane and, as a result, the nonlinear branch $k^{n \ell^{-}}$is seen to take over from $k_{r}^{\ell^{-}}$in a finite $X$-subinterval.

For frequencies such that the line $\omega=\omega_{c}$ in the $X-\omega$ plane intersects both regions $\mathcal{R}^{2^{ \pm}}(X, \omega)>0$, both linear branches cross the $k_{r}$-axis (fig.3-c1) and are connected to the corresponding nonlinear branches (fig.3-c2). Let $X_{1}^{ \pm}(\omega)$ and $X_{2}^{ \pm}(\omega)$ be the locations where $\mathcal{R}^{2^{ \pm}}(X, \omega)=0$, whereby $k^{\ell^{ \pm}}\left(X_{1,2}^{ \pm}\right)=k^{n \ell^{ \pm}}\left(X_{1,2}^{ \pm}\right)$. A nonlinear solution with local wavenumber $k^{n \ell^{ \pm}}(X, \omega)$ then exists in the range $X_{1}^{ \pm}<X<X_{2}^{ \pm}$. The locations $X_{1,2}^{ \pm}$depend on the frequency, and in $\S 6$ we show that, for any frequency, the linear and nonlinear solutions can be matched at all orders in $\epsilon$ through transition layers centered around $X_{1,2}^{ \pm}(\omega)$. Recall that boundary conditions (6) require keeping only the linear branch $k^{\ell^{ \pm}}(X)$ in the regions $X \rightarrow \pm \infty$ respectively. At the locations $X_{1}^{-}$and $X_{2}^{+}$where the linear branches $k^{\ell^{-}}$and $k^{\ell^{+}}$cross the $k_{r}$-axis they change over to the corresponding nonlinear branches $k^{n \ell^{-}}$and $k^{n \ell^{+}}$. Thus the nonlinear region is delimited by $X_{1}^{-}$ (where $k^{\ell^{-}}=k^{n \ell^{-}}$) and $X_{2}^{+}$(where $k^{n \ell^{+}}=k^{\ell^{+}}$), and a continuous global mode solution must necessarily connect both these nonlinear branches.

A smooth crossover between the nonlinear branches $k^{n \ell^{-}}$and $k^{n \ell^{+}}$(fig.3-s2) can only be achieved for frequencies such that the line $\omega=$ const. meets the critical curve limiting the allowable domain in the $X$ - $\omega$ plane. Furthermore, the line $\omega=$ const. has to be entirely contained within the allowable domain. Thus, the leading order global frequency $\omega_{s}$ is obtained by requiring that the line $\omega=\omega_{s}$ be tangent to the allowable domain in the $X$ - $\omega$ plane at a point $X_{s}$. Since, to each point in the allowable domain of the $X-\omega$ plane, correspond two pairs $\left(k^{n \ell^{+}}, \mathcal{R}^{2+}\right)$ and $\left(k^{n \ell^{-}}, \mathcal{R}^{2-}\right)$, the allowable domain may be considered as the superposition of two sheets respectively labelled $(+)$ and $(-)$ which necessarily join along the critical curve where $\left(k^{n \ell^{+}}, \mathcal{R}^{2^{+}}\right)=\left(k^{n \ell^{-}}, \mathcal{R}^{2^{-}}\right)$. A plane $\omega=$ const. cuts the surface made up of the two sheets along two lines, one on each sheet, which correspond to the $(+)$ and $(-)$ spatial branches respectively. Passing from one sheet to the other on such a line is possible only for $\omega=\omega_{s}$. The additional condition that the line $\omega=\omega_{s}$ be entirely contained in the allowable domain guarantees that the surface made up of the two sheets exhibits a saddle point when cut by the plane $\omega=\omega_{s}$. Thus, at leading order, the global frequency $\omega_{g}$ is given by a saddle point of the nonlinear dispersion relation. Moreover nonlinear global modes only exist if the saddle point is located in a region where the wavenumber $k_{s} \equiv k^{n \ell^{+}}\left(X_{s}, \omega_{s}\right)=$ $k^{n \ell^{-}}\left(X_{s}, \omega_{s}\right)$ is linearly unstable, i.e., $R_{s}^{2} \equiv \mathcal{R}^{2^{+}}\left(X_{s}, \omega_{s}\right)=\mathcal{R}^{2^{-}}\left(X_{s}, \omega_{s}\right)>0$.

An explicit condition for the existence of a saddle point may be derived from the 
local reduced nonlinear dispersion relation (24)

$$
\omega=\Omega^{N L}(k, X)
$$

This relation defines a surface in $(\omega, k, X)$ space which bears the same features as the complete nonlinear dispersion relation (23). For a given frequency $\omega$, the spatial branches $k^{n \ell^{ \pm}}(X, \omega)$ are obtained as the intersection of this surface and the plane $\omega=$ const. The condition that the branches $k^{n \ell^{ \pm}}(X)$ be connected, requires that the global frequency be given by a saddle point of the surface $\omega=\Omega^{N L}(k, X)$. Thus, the global frequency $\omega_{g}$ is obtained, at leading order in the WKBJ approximation, for a saddle point of the reduced nonlinear dispersion relation, i.e.,

$$
\omega_{g} \sim \omega_{s}=\Omega^{N L}\left(k_{s}, X_{s}\right)
$$

where the pair $\left(k_{s}, X_{s}\right)$ satisfies

$$
\frac{\partial \Omega^{N L}}{\partial k}\left(k_{s}, X_{s}\right)=\frac{\partial \Omega^{N L}}{\partial X}\left(k_{s}, X_{s}\right)=0
$$

The additional condition ${ }^{2}$

$$
\left(\Omega_{k X, s}^{N L}\right)^{2}-\Omega_{k k, s}^{N L} \Omega_{X X, s}^{N L}>0
$$

guarantees that $\left(\omega_{s}, k_{s}, X_{s}\right)$ is actually a saddle point and not a local extremum.

Criterion $(30,31)$ may also be expressed in terms of the complete dispersion relation $(23)$

$$
\omega=\Omega^{n \ell}\left(k, R^{2}, X\right) .
$$

Bearing in mind that

$$
\Omega^{N L}(k, X)=\Omega_{r}^{n \ell}\left(k, R^{2}(k, X), X\right)
$$

where $R^{2}(k, X)$ is implicitely defined by

$$
\Omega_{i}^{n \ell}\left(k, R^{2}(k, X), X\right)=0,
$$

\footnotetext{
${ }^{2}$ Throughout, subscript $(s)$ denotes evaluation at the saddle point $\left(k_{s}, R_{s}^{2}, X_{s}\right)$.
} 
one may relate the derivatives of $\Omega^{N L}$ to those of $\Omega^{n \ell}$. After elementary calculations, one finds that criterion (31) takes the alternate form

$$
\operatorname{Im}\left[\left(\Omega_{R^{2}, s}^{n \ell}\right)^{\star} \Omega_{k, s}^{n \ell}\right]=\operatorname{Im}\left[\left(\Omega_{R^{2}, s}^{n \ell}\right)^{\star} \Omega_{X, s}^{n \ell}\right]=0,
$$

i.e., the derivatives of the complex function $\Omega^{n \ell}$ with respect to each of its arguments have the same phase. Thus the leading order global frequency $\omega_{s}$ and the associated quantities $\left(k_{s}, R_{s}^{2}, X_{s}\right)$ are equivalently determined by

$$
\omega_{s}=\Omega^{n \ell}\left(k_{s}, R_{s}^{2}, X_{s}\right) \quad \text { and } \quad \operatorname{Arg} \Omega_{k, s}^{n \ell}=\operatorname{Arg} \Omega_{R_{, s}^{2}}^{n \ell}=\operatorname{Arg} \Omega_{X, s}^{n \ell} .
$$

Additional condition (32) may also be expressed in terms of $\Omega^{n \ell}$ after cumbersome but straightforward calculations.

The essential result of the present section is summarized by the saddle point conditions $(30,31)$ applicable to the reduced nonlinear dispersion relation $\omega=$ $\Omega^{N L}(k, X)$ : to leading order, the nonlinear global frequency $\omega_{s}$ satisfies a criterion that is formally identical to its linear counterpart $(1,2)$. However it should be emphasized that the linear criterion involves complex values of the wavenumber, frequency and spatial coordinate, whereas the nonlinear criterion only involves purely real values for these quantities.

It is possible to compute higher order corrections to the global frequency $\omega_{g}$ by a detailed analysis of the saddle point region (SP). As in the case of linear global modes with a double turning point $[6,11,16,20]$, it is found that its characteristic thickness is $O\left(\epsilon^{1 / 2}\right)$. Matching between the SP layer and the surrounding $\mathrm{CNL}^{ \pm}$regions leads to an expansion of the form

$$
\omega_{g} \sim \omega_{s}+\epsilon \omega_{1, n}
$$

where the $O(\epsilon)$ frequency correction term $\omega_{1, n}$ is parametrized by a non-negative integer $n$. Detailed calculations of this quantified set of fully nonlinear global modes are postponed to a future publication.

\section{Spatial Structure of Transition Layers $\mathbf{T L}^{1,2}$}

At the locations $X_{1}^{-}$and $X_{2}^{+}$where the amplitude of nonlinear wKBJ approximations vanishes, nonlinearities become weak, and, in the semi-infinite regions $\left(\mathrm{OL}^{ \pm}\right)$ extending towards $X= \pm \infty$, the solution is described by linear WKBJ approximations. The structure of the transition layer connecting the finite amplitude 
nonlinear solution in $\left(\mathrm{CNL}^{ \pm}\right)$to exponentially decaying linear solutions in $\left(\mathrm{OL}^{ \pm}\right)$ is analyzed in this section.

It is essential to bear in mind that this transition layer plays no rôle in the global frequency selection mechanism which solely depends on the local nonlinear dispersion relation at the saddle point $X_{s}$, in the core of the nonlinear region. The locations of the transition layers indeed depend on the frequency $\omega_{g}$ but the linear and nonlinear solutions can be matched for any frequency. For this reason matching is carried out for an arbitrary fixed real frequency $\omega$.

With the definitions of $\S 5$, the linear branch $k^{\ell^{-}}(X)$ meets the nonlinear branch $k^{n \ell^{-}}(X)$ at $X=X_{1}^{-}$. Let $k_{1} \equiv k^{\ell^{-}}\left(X_{1}^{-}\right)=k^{n \ell^{-}}\left(X_{1}^{-}\right)$be the local real wavenumber at ${ }^{3} X_{1}$. For $X<X_{1}$ the global mode is approximated by the linear WKBJ $\psi^{\ell}$ approximation (17) and for $X>X_{1}$ by the nonlinear wKBJ $\psi^{n \ell}$ approximation (26). None of these expressions are uniformly valid in a full neighborhood of $X_{1}$. An inner solution must then be constructed in an inner layer $\mathrm{TL}^{1}$ around $X_{1}$ to allow a smooth transition between both outer WKBJ approximations.

The form of the inner solution is obtained from the behavior of the outer solutions as $X \rightarrow X_{1}$. Near $X_{1}$ the nonlinear solution (26) expands into

$$
\psi^{n \ell}(X) \sim\left(X-X_{1}\right)^{1 / 2} \sqrt{\mathcal{R}_{X, 1}^{2}} e^{i \theta_{0}(X)} e^{i k_{1} x} \exp \left(\frac{i}{2 \epsilon} k_{X, 1}^{n \ell}\left(X-X_{1}\right)^{2}\right)
$$

with $^{4}$

$$
\mathcal{R}_{X, 1}^{2}=-\frac{\operatorname{Im}\left[\left(\Omega_{k, 1}^{n \ell}\right)^{\star} \Omega_{X, 1}^{n \ell}\right]}{\operatorname{Im}\left[\left(\Omega_{k, 1}^{n \ell}\right)^{\star} \Omega_{R^{2}, 1}^{n \ell}\right]} \quad \text { and } \quad k_{X, 1}^{n \ell}=-\frac{\operatorname{Im}\left[\left(\Omega_{R^{2}, 1}^{n \ell}\right)^{\star} \Omega_{X, 1}^{n \ell}\right]}{\operatorname{Im}\left[\left(\Omega_{R^{2}, 1}^{n \ell}\right)^{\star} \Omega_{k, 1}^{n \ell}\right]},
$$

and eq.(29) shows that

$$
\theta_{0}(X) \sim \frac{1}{2} \Theta_{0} \ln \left(X-X_{1}\right)+\text { const. } \quad \text { with } \quad \Theta_{0}=\frac{\operatorname{Re}\left[\left(\Omega_{R^{2}, 1}^{n \ell}\right)^{\star} \Omega_{k, 1}^{n \ell}\right]}{\operatorname{Im}\left[\left(\Omega_{R^{2}, 1}^{n \ell}\right)^{\star} \Omega_{k, 1}^{n \ell}\right]}
$$

while the linear solution displays the following asymptotic behavior

$$
\psi^{\ell}(X) \sim A_{0}^{\ell}\left(X_{1}\right) e^{i k_{1} x} \exp \left(\frac{i}{2 \epsilon} k_{X, 1}^{\ell}\left(X-X_{1}\right)^{2}\right)
$$

\footnotetext{
${ }^{3}$ For simplicity we drop from now on the superscript $(-)$.

${ }^{4}$ Subscript (1) always denotes evaluation at $k=k_{1}, R_{1}^{2}=0, X=X_{1}$. Note that $\Omega_{1}^{n \ell}=\Omega_{1}^{\ell}$ because $\mathcal{R}^{2}\left(X_{1}\right)=0$.
} 
with

$$
k_{X, 1}^{\ell}=-\frac{\Omega_{X, 1}^{\ell}}{\Omega_{k, 1}^{\ell}}
$$

The expansion of the outer solutions (33) and (36) shows that the inner solution proceeds in the form

$$
\tilde{\psi}(\tilde{X}) \sim \epsilon^{1 / 4}\left(\tilde{A}_{0}(\tilde{X})+\epsilon^{1 / 2} \tilde{A}_{1 / 2}(\tilde{X})+\ldots\right) e^{i k_{1} x}
$$

where $\tilde{X}=\left(X-X_{1}\right) / \epsilon^{1 / 2}$ is the inner local space variable in the $\mathrm{TL}^{1}$ layer.

From the governing equation for the inner solution $\tilde{\psi}$ in the transition layer

$$
\begin{gathered}
\omega\left(\tilde{A}_{0}+\epsilon^{\frac{1}{2}} \tilde{A}_{\frac{1}{2}}+\ldots\right)=\Omega^{n \ell}\left(k_{1}-i \epsilon^{\frac{1}{2}} \partial_{\tilde{X}}, \epsilon^{\frac{1}{2}}\left|\tilde{A}_{0}+\epsilon^{\frac{1}{2}} \tilde{A}_{\frac{1}{2}}+\ldots\right|^{2}, X_{1}+\epsilon^{\frac{1}{2}} \tilde{X}\right) \\
\left(\tilde{A}_{0}+\epsilon^{\frac{1}{2}} \tilde{A}_{\frac{1}{2}}+\ldots\right)
\end{gathered}
$$

we recover at lowest order

$$
\omega=\Omega^{n \ell}\left(k_{1}, 0, X_{1}\right)=\Omega^{\ell}\left(k_{1}, X_{1}\right) .
$$

At order $\epsilon^{1 / 2}$ equation (38) reduces to

$$
\left(\omega-\Omega_{1}^{\ell}\right) \tilde{A}_{1 / 2}=-i \Omega_{k, 1}^{n \ell} \frac{d \tilde{A}_{0}}{d \tilde{X}}+\Omega_{R^{2}, 1}^{n \ell}\left|\tilde{A}_{0}\right|^{2} \tilde{A}_{0}+\Omega_{X, 1}^{n \ell} \tilde{X} \tilde{A}_{0}
$$

Bearing in mind (39), this leads to a solvability condition for $\tilde{A}_{0}$

$$
i \Omega_{k, 1}^{n \ell} \frac{d \tilde{A}_{0}}{d \tilde{X}}=\left(\Omega_{X, 1}^{n \ell} \tilde{X}+\Omega_{R^{2}, 1}^{n \ell}\left|\tilde{A}_{0}\right|^{2}\right) \tilde{A}_{0}
$$

This equation is of the form

$$
\frac{d \tilde{A}_{0}}{d \tilde{X}}=\left(\left(a_{r}+i a_{i}\right) \tilde{X}+\left(b_{r}+i b_{i}\right)\left|\tilde{A}_{0}\right|^{2}\right) \tilde{A}_{0}
$$

and admits the solution

$$
\tilde{A}_{0}(\tilde{X})=\frac{\tilde{A}_{0}(0) \exp \left[\frac{a_{r}+i a_{i}}{2} \tilde{X}^{2}-\frac{i}{2} \frac{b_{i}}{b_{r}} \ln \left(1-2 b_{r}\left|\tilde{A}_{0}(0)\right|^{2} \int_{0}^{\tilde{X}} e^{a_{r} u^{2}} d u\right)\right]}{\sqrt{1-2 b_{r}\left|\tilde{A}_{0}(0)\right|^{2} \int_{0}^{\tilde{X}} e^{a_{r} u^{2}} d u}} .
$$


The inner solution $\tilde{\psi}$ in the $\mathrm{TL}^{1}$ layer has to be matched for $\tilde{X} \rightarrow \pm \infty$ with the outer solutions $\psi^{n \ell}$ and $\psi^{\ell}$ in the $\mathrm{CNL}^{-}$and $\mathrm{OL}^{-}$regions respectively. The square root behavior of $\psi^{n \ell}$ in $(33)$ requires that $\tilde{A}_{0}(\tilde{X})=O\left(\tilde{X}^{1 / 2}\right)$ as $\tilde{X} \rightarrow+\infty$. This implies that

$$
2 b_{r}\left|\tilde{A}_{0}(0)\right|^{2} \int_{0}^{\infty} e^{a_{r} u^{2}} d u=1 ; \quad \text { or } \quad\left|\tilde{A}_{0}(0)\right|^{2}=\frac{1}{b_{r}} \sqrt{\frac{-a_{r}}{\pi}} .
$$

With this value for $\left|\tilde{A}_{0}(0)\right|$ the inner solution (42) admits for $\tilde{X} \rightarrow+\infty$ the asymptotic expansion

$$
\tilde{A}_{0}(\tilde{X}) \sim \sqrt{-\frac{a_{r}}{b_{r}} \tilde{X}} \exp \frac{i}{2}\left[\left(a_{i}-\frac{b_{i}}{b_{r}} a_{r}\right) \tilde{X}^{2}+\frac{b_{i}}{b_{r}}(\ln \tilde{X}+\text { const. })\right] .
$$

Comparison of the coefficients in (40) and (41), and use of $(34,35)$ leads to

$$
-\frac{a_{r}}{b_{r}}=\mathcal{R}_{X, 1}^{2}, \quad a_{i}-\frac{b_{i}}{b_{r}} a_{r}=k_{X, 1}^{n \ell} \quad \text { and } \quad \frac{b_{i}}{b_{r}}=\Theta_{0} .
$$

Thus the choice of

$$
\tilde{A}_{0}(0)=\left(\frac{-a_{r}}{\pi b_{r}^{2}}\right)^{1 / 4}
$$

completely matches, at leading order, the inner solution $\tilde{\psi}$ given by (37) and (42) with the outer nonlinear solution $\psi^{n \ell}$ in the limit (33).

For this value of $\tilde{A}_{0}(0)$ the asymptotic behavior of the inner solution (42) as $\tilde{X} \rightarrow-\infty$ is

$$
\tilde{A}_{0}(\tilde{X}) \sim \frac{\tilde{A}_{0}(0)}{\sqrt{2}} \exp \left(\frac{1}{2}\left(a_{r}+i a_{i}\right) \tilde{X}^{2}+i \text { const. }\right) .
$$

As $a_{r}+i a_{i}=i k_{X, 1}^{\ell}$, the inner solution $\tilde{\psi}$ matches the outer linear WKBJ approximation $\psi^{\ell}$ given by (36) as long as

$$
\left|A_{0}^{\ell}\left(X_{1}\right)\right|=\epsilon^{1 / 4} \frac{\left|\tilde{A}_{0}(0)\right|}{\sqrt{2}} .
$$

This completes the proof that, for a given frequency, the linear and nonlinear WKBJ approximations can be matched at leading order through a transition layer 
of width $\epsilon^{1 / 2}$ (in terms of $X$ ). A similar analysis holds for the transition layer at $X_{2}^{+}$.

\section{Conclusions and Discussion}

A fully nonlinear frequency selection criterion has been derived for global modes on a doubly infinite interval, governed by the complex Ginzburg-Landau equation with spatially varying coefficients.

Let $\omega=\Omega^{N L}(k, X)$ denote the local reduced nonlinear dispersion relation obtained by eliminating the amplitude $R^{2}$ from the complete local nonlinear dispersion relation $\omega=\Omega^{n \ell}\left(k, R^{2}, X\right)$ pertaining to finite amplitude travelling waves. The global frequency $\omega_{g}$ on the doubly infinite interval is then, at leading order, a real saddle point $\omega_{s}$ of $\Omega^{N L}$ associated with the real wavenumber $k_{s}$ and station $X_{s}$ such that

$$
\omega_{g} \sim \omega_{s}=\Omega^{N L}\left(k_{s}, X_{s}\right) \text { and } \frac{\partial \Omega^{N L}}{\partial k}\left(k_{s}, X_{s}\right)=\frac{\partial \Omega^{N L}}{\partial X}\left(k_{s}, X_{s}\right)=0 .
$$

This saddle point condition is selected so as to ensure a smooth crossover between the two nonlinear spatial branches $k^{n \ell^{-}}$and $k^{n \ell^{+}}$present on either side of $X_{s}$.

The nonlinear global mode spatial structure is illustrated in fig.1. A spatially evolving complex $k^{\ell^{-}}$branch issuing from $X=-\infty$ and governed by the local linear dispersion relation $\omega=\Omega^{\ell}(k, X)$ in the $\mathrm{OL}^{-}$region experiences a transition to the fully nonlinear régime within a layer $\mathrm{TL}^{1}$ of thickness $O\left(\epsilon^{1 / 2}\right)$ centered around a point $X_{1}^{-}$of local neutral stability. In a "downstream" $\mathrm{CNL}^{-}$region of finite extent, the wave is fully nonlinear and its real wavenumber $k^{n \ell^{-}}$and amplitude $\mathcal{R}^{2^{-}}$ are governed by the local nonlinear dispersion relation $\omega=\Omega^{n \ell}\left(k, R^{2}, X\right)$. As the wave enters the SP layer centered around $X_{s}$, it is smoothly converted into a $k^{n \ell^{+}}$ spatial branch since condition (44) is satisfied. The $\mathrm{CNL}^{+}$region mirrors, for the $k^{n \ell^{+}}$wave, the $\mathrm{CNL}^{-}$region for the $k^{n \ell^{-}}$wave. A second transition layer $\mathrm{TL}^{2}$ centered around the locally neutral station $X_{2}^{+}$allows a return to a linear $k^{\ell^{+}}$ spatial wave in the $\mathrm{OL}^{+}$region, and a final exponential decay at $X=+\infty$.

These results should be compared with the corresponding double turning point linear frequency selection criterion derived in $[6,16,20]$. For infinitesimal waves governed by a local linear dispersion relation $\omega=\Omega^{\ell}(k, X)$ between complex wavenumber $k$ and frequency $\omega$, the complex global frequency $\omega_{g}$ is given by the saddle point condition $(1,2)$, where the frequency $\omega_{s}$, wavenumber $k_{s}$, and spatial coordinate $X_{s}$ are all complex. The linear global mode spatial structure is thus 
made up of complex $k^{\ell^{-}}$and $k^{\ell^{+}}$spatially evolving waves governed by the local linear dispersion relation $\omega=\Omega^{\ell}(k, X)$ in corresponding $\mathrm{OL}^{-}$and $\mathrm{OL}^{+}$regions near $X=\mp \infty$. The saddle point condition (2) ensures a smooth crossover from the $k^{\ell^{-}}$to the $k^{\ell^{+}}$branch in an inner region SP of thickness $O\left(\epsilon^{1 / 2}\right)$ centered around the complex saddle point $X_{s}$. In the linear case, the $\mathrm{TL}^{1,2}$ and $\mathrm{CNL}^{ \pm}$regions are naturally absent, but an inner SP layer structure is necessary in both linear and nonlinear studies. Indeed, both frequency selection criteria are formally identical. Paradoxically the nonlinear criterion involves only real variables and therefore appears simpler to implement, without any of the complexities associated with analytic continuation in the complex $X$-plane.

According to $[6,16]$, a necessary condition for the existence of a growing linear global mode is that the basic state be linearly absolutely unstable in a region of finite extent. According to the present study, a necessary condition for the existence of a self-sustained nonlinear gobal mode appears to be that the basic state only contain a linearly unstable region of finite extent corresponding to $\mathcal{R}^{2 \pm}>0$. Fully nonlinear global modes of the type shown here may in principle occur even if the medium is only convectively unstable.

The latter result is entirely consistent with the studies of Chomaz [4] and Couairon \& Chomaz [7]. In these recent investigations, nonlinear global modes on the semi-infinite interval $0<X<\infty$ and governed by the real Ginzburg-Landau equation with constant coefficients and both cubic and quintic nonlinearities have been examined from the dynamical systems point of view. In the supercritical case (stabilizing nonlinear terms), it is found that nonlinear global modes may exist even if the basic state is only linearly convectively unstable. Thus, there is clearly more and more evidence that the relation between local linear stability properties and global modes becomes somewhat tenous as the fully nonlinear régime is approached.

The recent numerical simulations of wakes in shallow layers performed by Schär \& Smith [22] underscore the importance of nonlinearities in the frequency selection process. When nonlinearities were turned off, the wake was found to oscillate at a Strouhal frequency $\omega \sim 0.17$, to be compared with $\omega_{s}=0.19$ resulting from the linear frequency selection criterion $(1,2)$. Upon retaining nonlinear terms, the "observed" wake frequency increased substantially to $\omega \sim 0.27$. The criterion proposed in the present study could possibly account for this discrepancy.

We wish to thank Stéphane Le Dizès for many stimulating discussions and his careful reading of the manuscript. We are particularly indebted to the gentle people of LadHyX for providing and maintaining a friendly research atmosphere. LadHyX is jointly supported by the École polytechnique and by the Engineering Science Directorate of the French National Center for Scientific Research (CNRS 
UMR-C0156). 


\section{References}

[1] C.M. Bender and S.A. Orszag, Advanced mathematical methods for scientists and engineers (McGraw-Hill, New York, 1978).

[2] A.J. Bernoff, Slowly varying fully nonlinear wavetrains in the Ginzburg-Landau equation, Physica D 30 (1988) 363.

[3] F.H. Busse, Transition to turbulence in Rayleigh-Bénard convection, in Hydrodynamic instabilities and the transition to turbulence, H.L. Swinney and J.P. Gollub, eds., (Springer, New York, 1985), 97.

[4] J.-M. Chomaz, Absolute and convective instabilities in nonlinear systems, Phys. Rev. Lett. 69 (1992) 1931.

[5] J.-M. Chomaz, P. Huerre and L.G. Redekopp, The effect of nonlinearity and forcing on global modes, in New Trends in nonlinear dynamics and pattern-forming phenomena, P. Coullet and P. Huerre, eds., (Plenum Press, New York, 1990), 259.

[6] J.-M. Chomaz, P. Huerre and L.G. Redekopp, A frequency selection criterion in spatially developing flows, Stud. Appl. Math. 84 (1991) 119.

[7] A. Couairon and J.-M. Chomaz, Absolute and convective instabilities, front velocities and global modes in nonlinear systems, in preparation.

[8] M.C. Cross and A.C. Newell, Convection patterns in large aspect ratio systems, Physica 10D (1984) 299.

[9] K. Hannemann and H. Oertel Jr, Numerical simulation of the absolutely and convectively unstable wake, J. Fluid Mech. 199 (1989) 55.

[10] L.N. Howard and N. Kopell, Slowly varying waves and shock structures in reactiondiffusion equations, Stud. Appl. Math. 56 (1977) 95.

[11] P. Huerre and P.A. Monkewitz, Local and global instabilities in spatially developing flows, Ann. Rev. Fluid Mech. 22 (1990) 473.

[12] R.E. Hunt and D.G. Crighton, Instability of flows in spatially developing media, Proc. R. Soc. Lond. A 435 (1991) 109.

[13] G.E. Karniadakis and G.S. Triantafyllou, Frequency selection and asymptotic states in laminar wakes, J. Fluid Mech. 199 (1989) 441.

[14] J. Kevorkian and J.D. Cole, Perturbation methods in applied mathematics (Springer, New York, 1981).

[15] Y. Kuramoto, Phase dynamics of weakly unstable periodic structures, Prog. Theor. Phys. 71 (1984) 1182.

[16] S. Le Dizès, P. Huerre, J.-M. Chomaz and P.A. Monkewitz, Linear global modes in spatially-developing media, Phil. Trans. R. Soc. Lond. A 354 (1996) 169. 
[17] P. Manneville, Structures dissipatives, chaos et turbulence (Aléa Saclay, 1991).

[18] C. Mathis, M. Provansal and L. Boyer, The Bénard-von Karman instability: an experimental study near the threshold, J. Phys. Paris Lett. 45 (1984) 483.

[19] P.A. Monkewitz, D.W. Bechert, B. Lehmann and B. Barsikow, Self-excited oscillations and mixing in heated round jets, J. Fluid Mech. 213 (1990) 611.

[20] P.A. Monkewitz, P. Huerre and J.M. Chomaz, Global linear stability analysis of weakly non-parallel shear flows, J. Fluid Mech. 251 (1993) 1.

[21] Y. Pomeau and P. Manneville, Stability and fluctuations of a spatially periodic convective flow, J. Phys. Lett 40 (1979) L-609.

[22] C. Schär and R.B. Smith, Shallow-water flow past isolated topography. Part II. Transition to vortex shedding. J. Atmos. Sci. 50 (1993) 1401.

[23] K.R. Sreenivasan, S. Raghu and D. Kyle, Absolute instability in variable density round jets, Exp. Fluids 7 (1989) 309.

[24] P.J. Strykowski and K.R. Sreenivasan, On the formation and suppression of vortex 'shedding' at low Reynolds numbers, J. Fluid Mech. 218 (1990) 71.

[25] P.J. Strykowski and D.L. Niccum, The stability of countercurrent mixing layers in circular jets, J. Fluid Mech. 227 (1991) 309. 